\title{
Polymerase chain reaction (PCR) diagnosis and identification of mucormycosis in patients with suspected invasive fungal infection
}

\author{
Nahid Gholinejad Ghadi ${ }^{1}$, Tahereh Shokohi ${ }^{2,3^{*}}$, Zahra Seifi ${ }^{1}$, Seyed Reza Aghili 2,3, Emmanuel \\ Roilides $^{4}$, Mehdi Nikkhah 5 , Rostam Poormosa ${ }^{5}$, Hossein Karami ${ }^{6}$, Laleh Vahedi Larjani ${ }^{\text {, }}$ Maryam \\ Ghasemi 7 , Imaan Haghani 2 \\ Student Research committee, School of Medicine, Mazandaran University of Medical Sciences, Sari, Iran. \\ Department of Medical Mycology, School of Medicine, Mazandaran University of Medical Sciences, Sari, Iran. \\ Invasive Fungi Research Centre (IFRC), Mazandaran University of Medical Sciences, Sari, Iran \\ Infectious Diseases Section, 3rd Department of Pediatrics, Faculty of Medicine, Aristotle University School of Health Sciences, Thessaloniki, Greece. \\ Department of Otolaryngology, School of Medicine, Mazandaran University of Medical Sciences, Sari, Iran. \\ Department of Pediatrics, School of Medicine, Mazandaran University of Medical Sciences, Sari, Iran. \\ Department of Pathology, school of Medicine, Mazandaran University of Medical Sciences, Sari, Iran.
}

Background:_Accurate diagnosis of mucormycosis, a lifethreatening fungal infection, remains a challenge for physicians.

Objectives: To identify the causative Mucorales in fresh clinical samples and formalin-fixed paraffin-embedded (FFPE) samples of patients with proven mucormycosis by using semi-nested PCR of Mucorales targeting 18S ribosomal DNA and sequencing.

Patients/Methods: All patients with proven mucormycosis according to the EORTC/MSG criteria admitted between 2015 and 2017 and histopathologically proven FFPE samples were included. PCR targeting the 18S rDNA of Mucorales and ITS region was performed and PCR products were then sequenced.

Results: From 2015 to 2017, 63 patients suspected to have invasive fungal infection and meeting the inclusion criteria were enrolled in our study. Based on direct microscopic examination and culture of fresh clinical specimens, 9 (14.3\%) cases of mucormycosis, 7 (11.1\%) cases of aspergillosis, 4 (6.3\%) cases of candidiasis and $1(1.6 \%)$ case of cryptococcosis were diagnosed. Based on the EORTC/MSG criteria for invasive fungal diseases, 9 mucormycosis cases were included as proven. Five of 9 (55.6\%) mucormycosis cases were confirmed by culture to be caused by Rhizopus species by standard phenotypic methods.

Overall, 27 cases with histological evidence of mucormycosis were found in this study. PCR assays were performed on DNA extracted from 18 FFPE samples, 4 fresh tissues and 5 grown colonies (Fig.1). Amplification of DNA yielded a band of approximately 400 base pairs (bp) by external primers (ZM1 and ZM2) in 11 cases and a band of approximately $170 \mathrm{bp}$ in gel electrophoresis by internal primers (ZM1 and ZM3) in 4 cases and by ITS primers in 4 cases.

The sequencing results for PCR product of 5 positive cultures were Rhizopus arrhizus (Fig. 1). PCR was also performed on 4 fresh tissues, in which direct microcopy exam was positive and culture did not grow. For these samples, sequencing results revealed Rhizopus arrhizus (Fig. 1). Among 18 samples of FFPE from the histopathologically proven cases of mucormycosis, 10 cases were Mucorales PCR positive. Among 9 fresh samples, 7 (77.8\%) Mucorales PCR (5 in $1^{\text {st }}$ and 2 in $2^{\text {nd }}$ PCR run) and 2 (22.2\%) ITS PCR were positive.

\begin{tabular}{lll}
\multicolumn{3}{l}{ Table 2. Underlying conditions predisposing to mucormycosis and mortality } \\
\hline Underlying condition & Number of patients (\%) & Number of patients (\%) who died \\
\hline Diabetes mellitus & $20(74.1)$ & $11(55.0)$ \\
\hline Neutropenia & $17(63.0)$ & $11(64.7)$ \\
\hline Use of immunosuppressive drugs & $7(25.9)$ & $5(71.4)$ \\
\hline Hematologic malignancies & $5(18.5)$ & $5(100.0)$ \\
\hline Organ transplant recipients & $1(3.7)$ & $0(0.0)$ \\
\hline
\end{tabular}

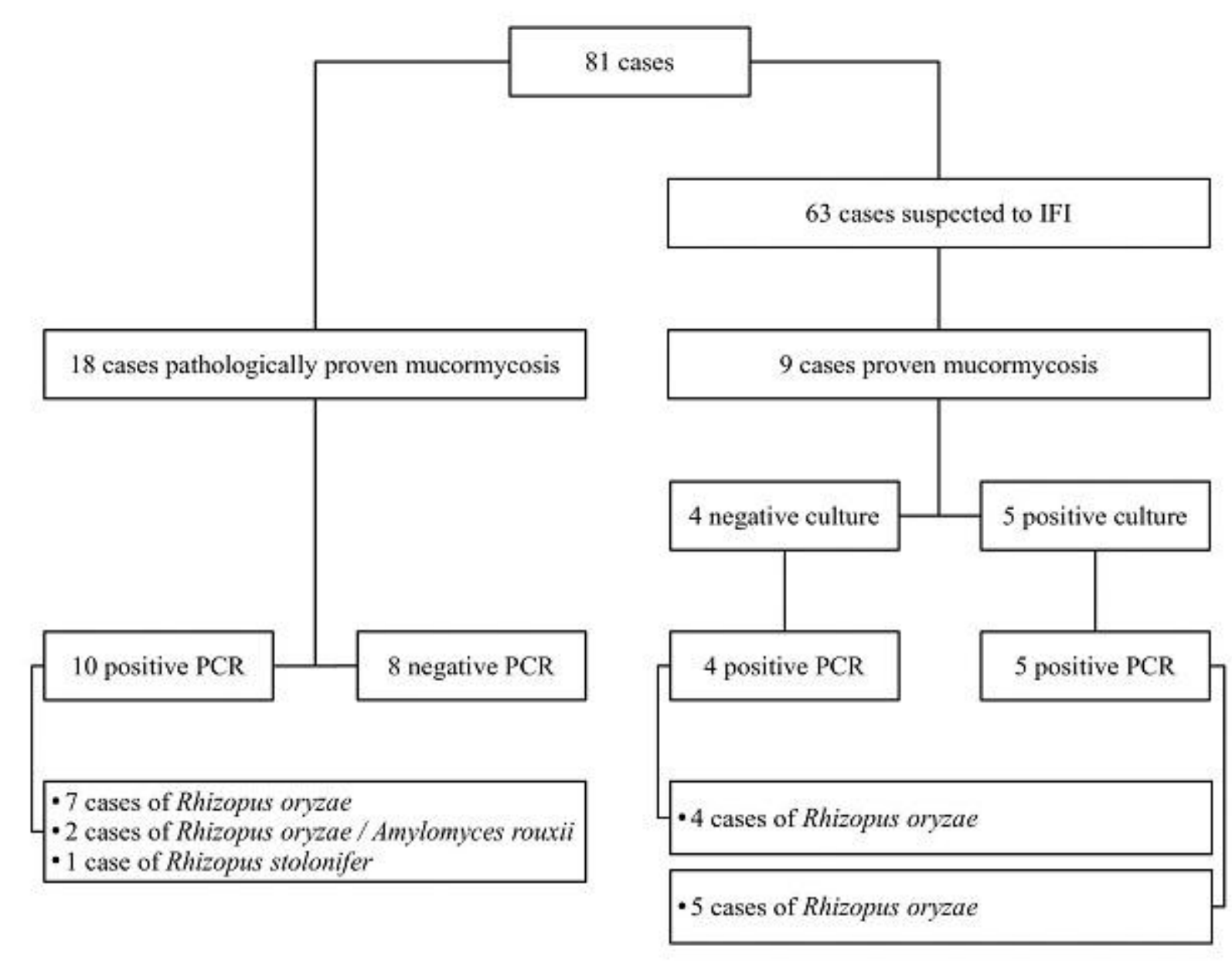

Figure 1: Mucormycosis diagnoses flow chart.

Overall, the species of Mucorales were Rhizopus arrhizus in 16 of 27 (59.3\%) cases, Rhizopus arrhizus / Amylomyces rouxii in 2 of 27 (7.4\%) cases and Rhizopus stolonifer in one of 27 (3.7\%) cases (Fig. 1 and Table 1). The Mucorales and ITS PCR were negative in eight of 27 (29.6\%) proven mucormycosis cases.

Among these cases, 7 (25.9\%) were male and 20 (74.1\%) were female, the mean age of the patients was $52.6 \pm 18.5$ years (range 4-78). Of these, 17 (63.0\%) patients died and 10 (37.0\%) patients survived. Clinical presentations of the patients with mucormycosis classified into rhinocerebral $(25 ; 92.6 \%)$ and disseminated (2; $7.4 \%$ ). Disseminated form is defined as the involvement of two or more noncontiguous organ systems. The most important predisposing factors for mucormycosis were diabetes mellitus (20; $74.1 \%$ ) and neutropenia in (17; 63.0\%) (Table 2). The mortality rate after 2 months of follow-up is indeed very high (63.0\%). All (5; $18.5 \%)$ patients with hematologic were died. 